\title{
On the Computation of Class Polynomials with "Thetanullwerte" and Its Applications to the Unit Group Computation
}

\author{
Franck Leprévost, Michael Pohst, and Osmanbey Uzunkol
}

\section{CONTENTS}

1. Introduction

2. Preliminaries

3. Class Polynomials

4. Class Units

5. Unit Group

6. Examples

Acknowledgments

References

2000 AMS Subject Classification: 11G15, 11Y35

Keywords: theta functions, complex multiplication, class fields
The classical class invariants of Weber are introduced as quotients of Thetanullwerte, enabling the computation of these invariants more efficiently than as quotients of values of the Dedekind $\eta$-function. We show also how to compute the unit group of suitable ring class fields by proving the fact that most of the invariants introduced by Weber are actually units in the corresponding ring class fields.

\section{INTRODUCTION}

Weber introduced the so-called Schläfli functions $\mathfrak{f}, \mathfrak{f}_{1}, \mathfrak{f}_{2}$ together with $\gamma_{2}$ and $\gamma_{3}$ in his Lehrbuch der Algebra [Weber 08] as quotients of values of the Dedekind $\eta$ function in order to generate the ring class field of an imaginary quadratic field $K$ with "simpler" generators than by $j$-invariants for a given order $\mathcal{O}_{t}$ of conductor $t \in \mathbb{Z}^{>0}$.

Another area in which the use of the Schläfli functions turns out to be more efficient than the use of the $j$-invariant is in the construction of special elliptic curves with a known number of rational points with the theory of complex multiplication, instead of taking a random elliptic curve over a finite field and computing the cardinality of rational points (see [Atkin and Morain 93, Morain 07] for applications in primality proving, and [Blake et al. 99, Blake et al. 05, Freeman et al. 06] for applications in group- and pairing-based cryptography). The interest comes from the fact that the minimal polynomials of the singular values of the class invariants, which are derived from the Schläfli functions, have smaller heights than the corresponding minimal polynomials of the singular values of the $j$-invariant.

In Section 2, we summarize some results from the theory of modular functions and the theory of theta functions that are to be used in the following sections. Also, modified Schläfli functions will be introduced as quotients of values of Jacobi theta functions, the so-called 
Thetanullwerte, and their relation to the classical Schläfli functions will be proved using a result from [Weber 08, pp. 112, 114].

In Section 3 we use the relationship between classical and modified Schläfli functions in order to express the class invariants as quotients of Thetanullwerte. Moreover, a complexity analysis of computing the Thetanullwerte and $\eta$ values, due to [Dupont 11], is given to show the efficiency of our method.

Using a theorem of Deuring, we prove in Section 4 the fact that most of these class invariants are units in the corresponding ring class fields. In case they are not units, we show that the absolute values of the norms of these invariants are powers $2^{l}$ with the property that the exponent $l$ divides the class number $h_{t}$. Furthermore, we prove that we obtain better class invariants in the cases $m \equiv 3 \bmod 24$ and $m=16 \cdot k+12$ for $k \equiv 3 \bmod 6$.

Using these class units, we will show in Section 5 how to compute the unit groups of the corresponding ring class fields.

Finally, the comparison of the computation of class polynomials using values of the $\eta$-function and Thetanullwerte and examples of the new class invariants are given in Section 6 as well as an application to the computation of the unit group of a ring class field.

\section{PRELIMINARIES}

We write as usual the discriminant function for $\tau \in \mathbb{H}=\{z \in \mathbb{C}: \Im(z)>0\}$ and the $j$-invariant at $\tau$ using Eisenstein series as follows (see for example [Deuring 58, p. 3]):

$$
\Delta(\tau):=g_{2}(\tau)^{3}-27 g_{3}(\tau)^{2}, j(\tau):=2^{6} 3^{3} g_{2}(\tau)^{3} \Delta(\tau)^{-1},
$$

And we define the Dedekind $\eta$-function by

$$
\eta(\tau)=q^{\frac{1}{24}} \prod_{k=1}^{\infty}\left(1-q^{k}\right) \text { with } q=\exp (2 \pi i \tau) .
$$

The theory of elliptic functions leads to the following identity (see [Deuring 58, p. 3]):

$$
\Delta(\tau)=(2 \pi)^{12} \eta(\tau)^{24}
$$

Weber's Schläfli functions can now be defined as quotients of values of the Dedekind $\eta$-function:

$$
\begin{aligned}
\mathfrak{f}(\tau) & =\exp \left(-\frac{\pi i}{24}\right) \frac{\eta\left(\frac{\tau+1}{2}\right)}{\eta(\tau)}, \quad \mathfrak{f}_{1}(\tau)=\frac{\eta\left(\frac{\tau}{2}\right)}{\eta(\tau)}, \\
\mathfrak{f}_{2}(\tau) & =\sqrt{2} \frac{\eta(2 \tau)}{\eta(\tau)}
\end{aligned}
$$

These functions satisfy the identities given in the following theorem [Weber 08, p. 114]:

Theorem 2.1. We have for all $\tau \in \nVdash$ the following identities:

(a) $\mathfrak{f}(\tau)^{8}=\mathfrak{f}_{1}(\tau)^{8}+\mathfrak{f}_{2}(\tau)^{8}$,

(b) $\mathfrak{f}(\tau) \mathfrak{f}_{1}(\tau) \mathfrak{f}_{2}(\tau)=\sqrt{2}$.

Lastly, the functions $\gamma_{2}$ and $\gamma_{3}$ are defined as follows:

$$
\gamma_{2}(\tau)=\sqrt[3]{j(\tau)}, \quad \gamma_{3}(\tau)=\sqrt{j(\tau)-12^{3}} .
$$

By [Schertz 02, p. 327], we have the following identities.

\section{Lemma 2.2.}

$$
\gamma_{2}=\frac{\mathfrak{f}^{24}-16}{\mathfrak{f}^{8}}=\frac{\mathfrak{f}_{1}^{24}+16}{\mathfrak{f}_{1}^{8}}=\frac{\mathfrak{f}_{2}^{24}+16}{\mathfrak{f}_{2}^{8}} .
$$

Definition 2.3. Let $\mathrm{H}_{g}$ denote the Siegel upper half-plane in dimension $g$ and let $\Omega \in \mathbb{H}_{g}$, i.e., $\Omega=\Omega_{1}+i \Omega_{2}$ with real $g \times g$ matrices $\Omega_{1}, \Omega_{2}$, whereby $\Omega_{2}$ is positive definite. The Riemann theta function is defined by

$$
\theta(z, \Omega)=\sum_{n \in \mathbb{Z}^{g}} \exp \left(\pi i\left(n^{t} \Omega n+2 n^{t} z\right)\right)
$$

for a column vector $z \in \mathbb{C}^{g}$. The theta characteristics for $\delta, \epsilon \in(\mathbb{Z} / 2 \mathbb{Z})^{g}$ are given by (see [Weng 01, p. 11])

$$
\begin{aligned}
\theta[\delta, \epsilon](z, \Omega) & \\
=\sum_{n \in \mathbb{Z}^{g}} \exp (\pi i & {\left[\left(n+\frac{1}{2} \delta\right)^{t} \Omega\left(n+\frac{1}{2} \delta\right)\right.} \\
+ & \left.\left.2\left(n+\frac{1}{2} \delta\right)^{t}\left(z+\frac{1}{2} \epsilon\right)\right]\right),
\end{aligned}
$$

from which it follows that

$$
\theta[\delta, \epsilon](-z, \Omega)=(-1)^{\delta^{t} \epsilon} \theta[\delta, \epsilon](z, \Omega) .
$$

If we set $z=0$, then we obtain the so-called Thetanullwerte (see [Weng 01, p. 11]).

Remark 2.4. The Thetanullwerte for $\delta^{t} \epsilon \equiv 1 \bmod 2$ are identically zero. These are called odd Thetanullwerte. If we have $\delta^{t} \epsilon \equiv 0 \bmod 2$, then we obtain even Thetanullwerte.

Hence there are $2^{g-1}\left(2^{g}+1\right)$ (respectively $2^{g-1}$ $\left.\left(2^{g}-1\right)\right)$ even (respectively odd) Thetanullwerte in $\mathfrak{H}_{g}$.

Definition 2.5. For $g=1, \tau \in \mathbb{H}$, and $q=\exp (2 \pi i \tau)$, the Thetanullwerte coincide with the classical Jacobi theta 
functions:

$$
\begin{aligned}
& \theta_{00}(\tau):=\theta[0,0](0, \tau)=\sum_{n \in \mathbb{Z}} q^{n^{2} / 2} \\
& \theta_{10}(\tau):=\theta[1,0](0, \tau)=\sum_{n \in \mathbb{Z}} q^{\left(n+\frac{1}{2}\right)^{2} / 2} \\
& \theta_{01}(\tau):=\theta[0,1](0, \tau)=\sum_{n \in \mathbb{Z}}(-1)^{n} q^{n^{2} / 2} \\
& \theta_{11}(\tau):=\theta[1,1](0, \tau)=\sum_{n \in \mathbb{Z}}(-1)^{n-\frac{1}{2}} q^{\left(n+\frac{1}{2}\right)^{2} / 2} .
\end{aligned}
$$

It is easy to see from Remark 2.4 that the Jacobi theta functions $\theta_{00}, \theta_{10}, \theta_{01}$ are even and $\theta_{11}$ is odd. We note that the derivative $\theta_{11}^{\prime}$ of $\theta_{11}$ is also an even function.

We now give the relationship between Schläfli functions and Jacobi theta functions according to the following theorem.

Theorem 2.6. [Weber 08, pp. 112, 114] For $\tau \in \mathbb{H}$, we have

$$
\begin{aligned}
& \theta_{11}^{\prime}(\tau)=2 \pi \eta(\tau)^{3} \\
& \theta_{00}(\tau)=\eta(\tau) \mathfrak{f}(\tau)^{2} \\
& \theta_{01}(\tau)=\eta(\tau) \mathfrak{f}_{1}(\tau)^{2} \\
& \theta_{10}(\tau)=\eta(\tau) \mathfrak{f}_{2}(\tau)^{2}
\end{aligned}
$$

Definition 2.7. For $\tau \in \mathbb{H}$, we introduce the following modified Schläfli functions:

$$
\begin{aligned}
\mathfrak{F}(\tau) & :=\frac{2 \theta_{00}(\tau)^{2}}{\theta_{01}(\tau) \theta_{10}(\tau)}, \\
\mathfrak{F}_{1}(\tau) & :=\frac{2 \theta_{01}(\tau)^{2}}{\theta_{00}(\tau) \theta_{10}(\tau)}, \\
\mathfrak{F}_{2}(\tau) & :=\frac{2 \theta_{10}(\tau)^{2}}{\theta_{00}(\tau) \theta_{01}(\tau)} .
\end{aligned}
$$

By Theorems 2.1 and 2.6, one can easily see the following relation:

$$
\eta(\tau)^{3}=\frac{\theta_{00}(\tau) \theta_{01}(\tau) \theta_{10}(\tau)}{2}
$$

Using this identity and the third powers of the identities in Theorem 2.6, we deduce the following theorem, which gives relations between classical and modified Schläfli functions.

Theorem 2.8. For $\tau \in \mathbb{H}$, we have the following:

$$
\begin{aligned}
\mathfrak{F}(\tau) & =\mathfrak{f}(\tau)^{6}, \\
\mathfrak{F}_{1}(\tau) & =\mathfrak{f}_{1}(\tau)^{6}, \\
\mathfrak{F}_{2}(\tau) & =\mathfrak{f}_{2}(\tau)^{6} .
\end{aligned}
$$

\section{CLASS POLYNOMIALS}

\subsection{Class Invariants and $\mathbf{N}$-Systems}

We refer to [Lang 73] for the basic properties of imaginary quadratic number fields, their orders, class field theory, modular functions, and the theory of complex multiplication.

Let $K$ be an imaginary quadratic number field with discriminant $d$, let $\mathcal{O}_{t}$ denote the order of $K$ of conductor $t \in \mathbb{Z}^{>0}$, and let $\mathrm{Cl}_{t}$ be the ring class group of $\mathcal{O}_{t}$ with class number $h_{t}$. We know from the theory of complex multiplication of imaginary quadratic number fields that for any number $\tau \in \mathbb{H}$ with discriminant $t^{2} d$, the value $j(\tau)$ generates the ring class field $\Omega_{t}$ of $K$, i.e., the field extension belonging to the subgroup $\mathcal{U}_{t}$ of the ideal group of $K$ generated by ideals of the form $(\lambda), \lambda \in \mathbb{Z}$, with $\operatorname{gcd}(\lambda, t)=1$ and $\lambda \equiv r \bmod t$ for a suitable $r \in \mathbb{Z}$; see [Schertz 02, p. 327].

The values $j\left(\tau_{i}\right), i=1, \ldots, h_{t}$, from the representatives of ideals $\left[\tau_{i}, 1\right]$ in $\mathrm{Cl}_{t}$ with $\tau:=\tau_{1}$ form a complete system of conjugate numbers over $K$. Furthermore, it is even a complete system of conjugates over $\mathbb{Q}$ [Lang 73 , p. 133, Remark 1]. Since the $j(\tau)$ for all $\tau \in \mathbb{H}$ are algebraic integers, the minimal polynomial of $j(\tau)$ has coefficients in $\mathbb{Z}$. We have the property that $\mathbb{Q}(j(\tau))$ is the conjugate field to the maximal real subfield of $\Omega_{t}$ (see [Schertz 76, p. 51]):

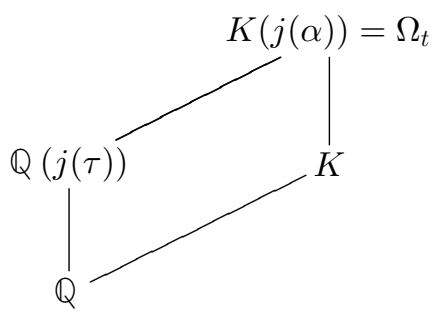

Let $g$ be one of the Schläfli functions $\mathfrak{f}, \mathfrak{f}_{1}, \mathfrak{f}_{2}$ or $\gamma_{2}, \gamma_{3}$. Then according to Lemma 2.2, we have

$$
\mathbb{Q}(j(\tau)) \subseteq \mathbb{Q}(g(\tau)) .
$$

If the other inclusion holds as well, for example for a small power of one of the values of Schläfli functions, we get an alternative primitive element for the field $\Omega_{t}$.

Definition 3.1. A value $g(\tau)$ of a modular function $g$ is said to be a class invariant if $\mathbb{Q}(g(\tau))=\mathbb{Q}(j(\tau))$.

In order to be able to generate the field $\mathbb{Q}(j(\tau))$ with some class invariant, we need to describe the conjugates of the invariant, which is possible according to the following definitions [Schertz 02, p. 329] and theorem. 
Definition 3.2. An imaginary quadratic integer $\tau \in H \cap K$ is the zero of a quadratic equation of the form $A x^{2}+B x+C=0$, which is uniquely determined by $\tau$, if we postulate the following normalization assumption:

$$
A, B, C \in \mathbb{Z}, \quad \operatorname{gcd}(A, B, C)=1, \quad A>0 .
$$

Such an equation is called primitive.

Definition 3.3. Let $N \in \mathbb{Z}^{>0}$ and $\tau_{1}, \tau_{2}, \ldots, \tau_{h_{t}} \in \mathbb{H}$, so that

$$
\left[\tau_{1}, 1\right],\left[\tau_{2}, 1\right], \ldots,\left[\tau_{h_{t}}, 1\right]
$$

is a system of representatives of $\mathrm{Cl}_{t}$. Let further $A_{i} x^{2}+$ $B_{i} x+C_{i}=0$ be primitive equations for $\tau_{i}$ that satisfy the properties

$\operatorname{gcd}\left(A_{i}, N\right)=1 \quad$ and $\quad B_{i} \equiv B_{j} \bmod 2 N, 1 \leq i, j \leq h_{t}$.

Then the elements $\tau_{1}, \tau_{2}, \ldots, \tau_{h_{t}}$ are called an $N$ system modulo $t$.

Remark 3.4. According to [Schertz 02, p. 335], we know that there exists an $N$-system for every natural number in $\mathbb{Z}^{>0}$.

We have the following theorem, which allows us to compute the class invariants as quotients of Thetanullwerte instead of computing them traditionally as values of quotients of the Dedekind $\eta$-function.

Theorem 3.5. Let $\tau \in \mathbb{H}$ be a zero of a primitive equation $A x^{2}+B x+C=0 \quad$ with $\operatorname{gcd}(A, 2)=1, B \equiv 0 \bmod 32$ with the special discriminant $D(\tau)=t^{2} d=:-4 m$, i.e., $D(\tau)$ is divisible by 4 with cofactor $-m$. Then the following numbers $g(\tau)$ are class invariants:

- $\left(\frac{2}{A}\right) \frac{1}{2 \sqrt{2}} \mathfrak{F}(\tau)=\left(\left(\frac{2}{A}\right) \frac{1}{\sqrt{2}} \mathfrak{f}(\tau)^{2}\right)^{3}$ if $m \equiv 1 \bmod 8$,

- $\exp \left(-\frac{\pi i}{8}\right) \frac{\theta_{11}^{\prime}\left(\frac{\tau+1}{2}\right)}{\theta_{11}^{\prime}(\tau)}=\mathfrak{f}(\tau)^{3}$ if $m \equiv 3 \bmod 8$,

- $\frac{\mathfrak{F}(\tau)^{2}}{8}=\left(\frac{1}{2} \mathfrak{f}(\tau)^{4}\right)^{3}$ if $m \equiv 5 \bmod 8$,

- $\left(\frac{2}{A}\right) \frac{1}{2 \sqrt{2}} \exp \left(-\frac{\pi i}{8}\right) \frac{\theta_{11}^{\prime}\left(\frac{\tau+1}{2}\right)}{\theta_{11}^{\prime}(\tau)}=\left(\left(\frac{2}{A}\right) \frac{1}{\sqrt{2}} \mathfrak{f}(\tau)\right)^{3}$ if $m \equiv 7 \bmod 8$,

- $\left(\frac{2}{A}\right) \frac{1}{2 \sqrt{2}} \mathfrak{F}_{1}(\tau)=\left(\left(\frac{2}{A}\right) \frac{1}{\sqrt{2}} \mathfrak{f}_{1}(\tau)^{2}\right)^{3}$ if $m \equiv 2 \bmod 4$,

- $\left(\frac{2}{A}\right) \frac{\mathfrak{F}_{1}(\tau)^{2}}{16 \sqrt{2}}=\left(\left(\frac{2}{A}\right) \frac{1}{2 \sqrt{2}} \mathfrak{f}_{1}(\tau)^{4}\right)^{3}$ if $m \equiv 4 \bmod 8$,

where $\left(\frac{2}{A}\right)$ denotes the Legendre symbol.

If $\tau=\tau_{1}, \ldots, \tau_{h_{t}}$ is a 16-system modulo $t$, then the singular values $g\left(\tau_{i}\right)$ above form a complete system of conjugates over $\mathbb{Q}$. Therefore, the minimal polynomial over $\mathbb{Q}$ is

$$
W_{D(\tau)}(x)=\prod_{i=1}^{h_{t}}\left(x-g_{i}\right),
$$

where $g_{i}:=g\left(\tau_{i}\right)$, and this polynomial has integer coefficients; $W_{D(\tau)}(x)$ is called the class polynomial of $g(\tau)$.

Proof: The identities between the quotients of values of the Dedekind $\eta$-function and the quotients of the Thetanullwerte follow from Theorems 2.8 and 2.6. The result for quotients of the values of the Dedekind $\eta$ function is a theorem of Schertz [Schertz 02, p. 337].

Remark 3.6. The values in Theorem 3.5 are the elements of $\Omega_{t}$ without the factor $\left(\frac{2}{A}\right)$. This factor is required only for writing down the conjugates for $g(\tau)$; see [Schertz 02] for details.

For discriminants not divisible by 3 , the functions in Theorem 3.5 without outer exponent 3 are also class invariants [Schertz 02, p. 330, Theorem 2]. This follows from the relation between $\mathfrak{f}$ and $\gamma_{2}$ in Lemma 2.2.

\subsection{Optimality: The Choice of Class Invariant}

The natural questions as to which invariants should be used in practice and how well class invariants can be constructed using alternative modular functions will be discussed in this section.

Let $g$ be a modular function whose value $g(\tau)$ is a class invariant. The logarithmic height of the minimal polynomial of $g(\tau)$ differs from the logarithmic height of $j(\tau)$ by a constant factor according to the following theorem of Hindry and Silverman.

Theorem 3.7. [Hindry and Silverman 00, Proposition B.3.5] Let $r(g)$ be the quotient

$$
r(g)=\frac{\operatorname{deg}_{g}(\Phi(g, j))}{\operatorname{deg}_{j}(\Phi(g, j))},
$$

where $\Phi(g, j)=0$ is the modular polynomial and $\operatorname{deg}_{x}(\Phi(x, y)$ the degree in $x$ of this polynomial.

Then, we have

$$
r(g)=\lim _{\mathcal{H}(j(\tau)) \rightarrow \infty} \frac{\mathcal{H}(g(\tau))}{\mathcal{H}(j(\tau))},
$$

where the limit is taken over all CM-points $\tau \in \mathbb{H}$, which are ordered by the discriminant of the corresponding orders, and $\mathcal{H}$ is the absolute logarithmic height.

Bröker and Stevenhagen proved the following theorem using Theorem 3.7. 
Theorem 3.8. [Bröker and Stevenhagen 08.] We have the upper bound

$$
r(g) \leq 32768 / 325 \approx 100.82 .
$$

Assume that Selberg's eigenvalue conjecture holds (see [Sarnak 95]). Then we have

$$
r(g) \leq 96 .
$$

Using the definition of $r(g)$ together with Theorem 3.5, Lemma 2.2, and Remark 3.6, we obtain the following theorem. It states which constant factor can be gained using the class invariants of Theorem 3.5.

Theorem 3.9. Let the assumptions be as in Theorem 3.5. Then we have

$$
r(g)= \begin{cases}6 & \text { if } m \equiv 12,21 \bmod 24 \\ 12 & \text { if } m \equiv 6,9,18 \bmod 24 \\ 18 & \text { if } m \equiv 4,5,13,20 \bmod 24 \\ 24 & \text { if } m \equiv 3,15 \bmod 24 \\ 36 & \text { if } m \equiv 1,2,10,14,17,22 \bmod 24 \\ 72 & \text { if } m \equiv 7,11,19,23 \bmod 24\end{cases}
$$

By Theorems 3.9 and 3.6, we obtain $r(g)=72$ for class invariants $g(\tau)=\mathfrak{f}(\tau)$ and $g(\tau)=\frac{1}{\sqrt{2}} \mathfrak{f}(\tau)$ in the cases $m \equiv 3 \bmod 8$ and $m \equiv 7 \bmod 8$, respectively, if the discriminant is not divisible by 3 . Hence, these are almost optimal class invariants.

It is an open question whether there is a modular function $g$ with $r(g)=96$ whose suitable values are class invariants.

\subsection{Analysis: $\theta$ versus $\eta$}

An asymptotically fast algorithm for the numerical computation of the $n$ significant bits of one of the Thetanullwerte evaluated at $\tau \in \mathbb{H}$ is given in [Dupont 11]. The author uses the connection between arithmetic-geometric means (AGM) of complex numbers and Thetanullwerte. He proved that the computation can be done in $\mathcal{O}(M(n) \log n)$ bit operations, where $M(n)$ denotes the time complexity of multiplying two $n$-bit integers. We explain now how one can compute the $n$ significant bits of a value of the Dedekind $\eta$-function using his algorithm.

Definition 3.10. We define $\kappa$ and $\kappa^{\prime}$ for $\tau \in \mathbb{H}$ as follows:

$$
\kappa(\tau)=\left(\frac{\theta_{10}(\tau)}{\theta_{00}(\tau)}\right)^{2}, \quad \kappa^{\prime}(\tau)=\left(\frac{\theta_{01}(\tau)}{\theta_{00}(\tau)}\right)^{2} .
$$

Theorem 3.11. Using the identity

$$
\eta(\tau)^{12}=\frac{\kappa^{\prime}(\tau)^{2}\left(1-\kappa^{\prime}(\tau)^{2}\right) \theta_{00}(\tau)^{12}}{16},
$$

one can compute $\eta(\tau)^{12}$ in $O(\mathcal{M}(n) \log n)$ bit operations.

Proof: By [Dupont 11, p. 1844], we have

$$
\mathfrak{f}(\tau)^{24} \kappa^{\prime}(\tau)^{2}\left(1-\kappa^{\prime}(\tau)^{2}\right)=16 .
$$

By Theorem 2.6, we have $\mathfrak{f}(\tau)^{24} \eta(\tau)^{12}=\theta_{00}(\tau)^{12}$. This implies

$$
\eta(\tau)^{12}=\frac{\kappa^{\prime}(\tau)^{2}\left(1-\kappa^{\prime}(\tau)^{2}\right) \theta_{00}(\tau)^{12}}{16} .
$$

Since one can compute the $n$ significant bits of one of the Thetanullwerte evaluated at $\tau \in \mathbb{H}$ in $\mathcal{O}(M(n) \log n)$, we can compute $\eta(\tau)^{12}$ also in $\mathcal{O}(M(n) \log n)$.

Note that the same arguments for computing $\eta(\tau)^{12}$ are given in [Dupont 11, p. 1844]. However, the power of $\theta_{00}(\tau)$ on line 3 from the bottom of page 1844 is not stated correctly.

Therefore, we need to extract the 12 th root of $\eta^{12}$ in order to evaluate $n$ significant bits of the $\eta$-function evaluated at $\tau$, which can be done by means of Newton iteration. This computation has the complexity $\mathcal{O}(M(n))$. Hence, if we compute the class invariants as quotients of Thetanullwerte, we save the time needed to take a 12 th root using Newton iteration. Note that in taking a 12th root, no precision is lost in general; see [Dupont 11, p. 1827]. This means that the precision we need is the same in both cases. We save $\mathcal{O}(M(n))$ bit operations using the Thetanullwerte and avoiding Newton iteration.

Note also that in order to compute a value $\eta(\tau)$ using Theorem 3.11, we need to compute both $\theta_{00}(\tau)$ and $\theta_{01}(\tau)$. Hence, we need approximately twice as many coefficients in the computation of $\eta(\tau)$ as in that of $\theta_{00}(\tau)$ or $\theta_{01}(\tau)$.

We refer to the last section for a comparison of computing class polynomials using $\eta$ and $\theta$ representations.

Another reason to use the quotients of Thetanullwerte instead of the values of quotients of the Dedekind $\eta$-function is that Thetanullwerte are functions that can be generalized to any genus. Since there is no analogue of the notion of smaller class invariants for genus greater than 1 , the representation of class invariants as quotients of Thetanullwerte can be used to generalize the notion of class invariants at least to genus two; see [Uzunkol 10, p. 117]. 


\section{CLASS UNITS}

In this section, we prove that most of the invariants introduced by the above theorems are actually units in the corresponding ring class fields. This enables us to compute the unit group of ring class fields using these explicit units, which will be discussed in the next section. We begin with a theorem of Deuring. (Note that although some of the results given in this section are stated in [Birch 69], proofs are not given.)

Let $P$ be a primitive matrix of determinant $p$, where $p$ is a prime number, i.e.,

$$
P=\left(\begin{array}{ll}
a & b \\
c & d
\end{array}\right) \in \mathbb{Z}^{2 \times 2}
$$

with $\operatorname{det}(P)=p$ and $\operatorname{gcd}(a, b, c, d)=1$.

For the quotient (see [Deuring 58, p. 11])

$$
\varphi_{P}(\tau):=p^{12} \frac{\Delta\left(P\left(\begin{array}{l}
\omega_{1} \\
\omega_{2}
\end{array}\right)\right)}{\Delta\left(\begin{array}{l}
\omega_{1} \\
\omega_{2}
\end{array}\right)},
$$

where

$$
\Delta\left(\begin{array}{l}
\omega_{1} \\
\omega_{2}
\end{array}\right)=\omega_{2}^{-12} \Delta(\tau)
$$

we have the following theorem of Deuring.

Theorem 4.1. [Deuring 58, p. 43] Let $t>0$ be an integer, $p$ a prime number, and $l \geq 0$ the greatest power of $p$ such that $p^{l} \mid t$. Let further $a, b, c, d$ be integers such that the matrix

$$
P:=\left(\begin{array}{ll}
a & b \\
c & d
\end{array}\right)
$$

has determinant $p$. Assume that $\left\{\omega_{1}, \omega_{2}\right\}$ is a basis of a fractional $\mathcal{O}_{t}$-ideal $I$ with $\tau:=\frac{\omega_{1}}{\omega_{2}} \in \mathbb{H}$. Then we have the following:

1. If $p$ splits completely in $K$, i.e., $(p)=\mathfrak{p} \overline{\mathfrak{p}}$, then

(a) $\varphi_{P}(\tau)$ is a unit if $P\left(\begin{array}{l}\omega_{1} \\ \omega_{2}\end{array}\right)$ is a basis of a fractional $\mathcal{O}_{t p}$-ideal;

(b) $\frac{\varphi_{P}(\tau)}{p^{12}}$ is a unit if $P\left(\begin{array}{c}\omega_{1} \\ \omega_{2}\end{array}\right)$ is a basis of a fractional $\mathcal{O}_{t p^{-1}}-i d e a l$;

(c) In the case $l=0, \frac{\varphi_{P}(\tau)}{\overline{\mathfrak{p}}^{12}}$ and $\frac{\varphi_{P}(\tau)}{\mathfrak{p}^{12}}$ are units if $P\left(\begin{array}{l}\omega_{1} \\ \omega_{2}\end{array}\right)$ is a basis of ideals $I_{\mathcal{O}_{t}} \mathfrak{p}_{\mathcal{O}_{t}}$ and $I_{\mathcal{O}_{t}} \overline{\mathfrak{p}}_{\mathcal{O}_{t}}$, respectively.

2. If $p$ ramifies in $K$, i.e., $(p)=\mathfrak{p}^{2}$, then

(a) $\frac{\varphi_{P}(\tau)}{p^{\frac{6}{p^{l+1}}}}$ is a unit if $P\left(\begin{array}{c}\omega_{1} \\ \omega_{2}\end{array}\right)$ is a basis of a fractional $\mathcal{O}_{t p}-i d e a l$ (b) $\frac{\varphi_{P}(\alpha)}{12-\frac{6}{p^{l}}}$ is a unit if $P\left(\begin{array}{c}\omega_{1} \\ \omega_{2}\end{array}\right)$ is a basis of a fractional $\mathcal{O}_{t p^{-1}}-i d e a l$

(c) $\frac{\varphi_{P}(\tau)}{p^{6}}$ is a unit if $P\left(\begin{array}{l}\omega_{1} \\ \omega_{2}\end{array}\right)$ is a basis of the ideal
$I_{\mathcal{O}_{t}} \mathfrak{p}_{\mathcal{O}_{t}}$.

3. If $p$ is inert in $K$, i.e., $(p)=\mathfrak{p}$, then

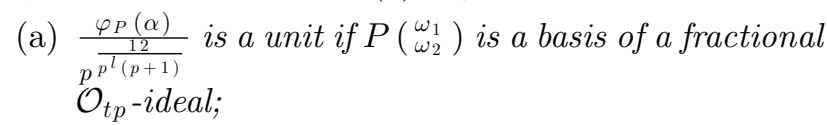

(b) $\frac{\varphi_{P}(\alpha)}{{ }_{p}^{12}\left[1-\frac{1}{p^{l-1}(p+1)}\right]}$ is a unit if $P\left(\begin{array}{c}\omega_{1} \\ \omega_{2}\end{array}\right)$ is a basis of a fractional $\mathcal{O}_{t p^{-1}}-$ ideal.

Theorem 4.2. Let $g(\tau)$ be the class invariants as in Theorem 3.5. Then $g(\tau)$ is a unit if $m \equiv 1,5,7 \bmod 8$ or $m \equiv 2 \bmod 4$, where $D(\tau)=-4 m$.

Proof: We will prove the theorem using equations (2-1) and $(2-2)$ in each case.

Let $\tau \in \mathbb{Q}(\sqrt{d})=\mathbb{Q}\left(\sqrt{d^{\prime}}\right)=: K$ with $\Delta(\tau)=-4 m=t^{2} d=t^{\prime 2} d^{\prime}$, where $d^{\prime}$ is square-free.

In the cases $m \equiv 1,5 \bmod 8$, or equivalently $m \equiv 1 \bmod 4$, we have $t^{\prime} \equiv 0 \bmod 2$, for otherwise $d^{\prime}$ would not be a square-free integer. Letting $t^{\prime}=2 s$ yields $s^{2} d^{\prime} \equiv-1 \bmod 4$, which shows that $s$ must be an odd integer and hence $s^{2} \equiv 1 \bmod 4$ and $d^{\prime} \equiv-1 \bmod 4$.

Hence for $m \equiv 1 \bmod 4$, we have $\mathcal{O}_{K}=\mathbb{Z}\left[\sqrt{d^{\prime}}\right]$ and $(2)=\mathfrak{p}^{2}$, since 2 ramifies in $\mathcal{O}_{K}$. Considering the basis $\{\tau, 1\}$ of $\mathcal{O}_{t}$ together with the matrix

$$
P=\left(\begin{array}{ll}
1 & t \\
0 & 2
\end{array}\right)
$$

we have

$$
P\left(\begin{array}{l}
\tau \\
1
\end{array}\right)=[\tau+t, 2]
$$

as a basis of the ideal $\mathfrak{p} \mathcal{O}_{t}$.

Applying the result of Theorem 4.1 (2(c)), we have the property that $\frac{\varphi_{P}(\tau)}{2^{6}}$ is a unit, which means that

$$
2^{-6} \frac{\Delta\left(\frac{\tau+1}{2}\right)}{\Delta(\tau)}
$$

is a unit.

For $m \equiv 1 \bmod 8$, we obtain by $(2-1)$ and $(2-2)$ that

$$
2^{-6} \frac{\Delta\left(\frac{\tau+1}{2}\right)}{\Delta(\tau)}=g(\tau)^{4}=\left(\left(\left(\frac{2}{A}\right) \frac{\mathfrak{f}(\tau)^{2}}{\sqrt{2}}\right)^{3}\right)^{4},
$$

which shows that the invariant $g(\tau)$ is a unit. 
For $m \equiv 5 \bmod 8$, we obtain similarly

$$
2^{-6} \frac{\Delta\left(\frac{\tau+1}{2}\right)}{\Delta(\tau)}=g(\tau)^{2}=\left(\left(\frac{\mathfrak{f}(\tau)^{4}}{2}\right)^{3}\right)^{2},
$$

which shows that the invariant $g(\tau)$ is a unit.

In the case $m \equiv 7 \bmod 8$, using a similar argument as above, we have $t^{\prime} \equiv 0 \bmod 2,-d^{\prime} \equiv 3 \bmod 4$, $\mathcal{O}_{K}=\mathbb{Z}\left[\frac{1+\sqrt{d^{\prime}}}{2}\right]$, and 2 splits completely in $\mathcal{O}_{K}$. Using Theorem $4.1(1(\mathrm{~b}))$ with the basis $\{\tau+t, 1\}$ of an $\mathcal{O}_{2 t^{-}}$ ideal and

$$
P=\left(\begin{array}{ll}
1 & 0 \\
0 & 2
\end{array}\right),
$$

we have,

$$
\frac{\varphi_{P}(\tau+t)}{2^{12}}=g(\tau)^{8}=\left(\left(\left(\frac{2}{A}\right) \frac{\mathfrak{f}(\tau)}{\sqrt{2}}\right)^{3}\right)^{8}
$$

is a unit, which shows that the invariant $g(\tau)$ is also a unit in this case.

In the last case, $m \equiv 2 \bmod 4$, we have $t \equiv 1 \bmod 2$, $d^{\prime} \equiv 2 \bmod 4, \mathcal{O}_{K}=\mathbb{Z}[\sqrt{d}]$, and 2 ramifies in $\mathcal{O}_{K}$. Using Theorem $4.1(2(\mathrm{c}))$ again as in the first case but with an odd $t$ yields that

$$
2^{-6} \frac{\Delta\left(\frac{\tau}{2}\right)}{\Delta(\tau)}=g(\tau)^{4}=\left(\left(\left(\frac{2}{A}\right) \frac{\mathfrak{f}_{1}(\tau)^{2}}{\sqrt{2}}\right)^{3}\right)^{4}
$$

is a unit, which shows that the invariant $g(\tau)$ is also a unit.

Remark 4.3. According to Theorem 3.5, the invariants in Theorem 4.2 are units in the corresponding ring class fields.

For other cases, i.e., $m \equiv 3 \bmod 8$ and $m \equiv 12 \bmod 16$, we know that the invariants are not units in the ring class field. However, we use Theorem 4.1 to show that there are units related to these invariants; hence we are going to show that the constant coefficients of minimal polynomials of these invariants are all powers of 2 , say $2^{l}$, with $l \mid h$.

We are going to show also that in the cases $m \equiv 3 \bmod 24$ and $m \equiv 4 \bmod 16$, we can get better class invariants. Furthermore, it will be shown that in the case $m \equiv 4 \bmod 16$, the invariant given in Theorem 3.5 is also a unit in the ring class field.

Theorem 4.4. Let $g(\tau)$ be the class invariant as in Theorem 3.5 and $h_{t}$ the class number of the discriminant of $\tau$. Then we have the following:

1. For $m \equiv 3 \bmod 8$ : (a) $\tilde{g}(\tau):=g(\tau) / 2$ is a class invariant and a unit if $m \equiv 3 \bmod 24$,

(b) $g(\tau)$ has the norm $2^{l}$ with $h_{t}=3 l$ if $m \equiv 11,19 \bmod 24$.

2. For $m \equiv 4 \bmod 8$ :

(a) $g(\tau)$ is a unit if $m \equiv 4 \bmod 16$;

(b) for $m \equiv 4 \bmod 16$, we write $m=16 k+12$, and then we have:

- $g(\tau)$ has the norm $2^{l}$ with $h_{t}=2 l$ if $k \equiv 0,1,5 \bmod 6$,

- $g(\tau)$ has the norm $2^{l}$ with $h_{t}=6 l$ if $k \equiv 2,4 \bmod 6$,

- $\tilde{g}(\tau):=g(\tau) / 2$ is a class invariant with norm $2^{l}$ and $h_{t}=2 l$ if $k \equiv 3 \bmod 6$.

Proof: For $m \equiv 3 \bmod 8$, we obtain with a similar argument as in the proof of Theorem $4.2, d=d^{\prime} \equiv 5 \bmod 8$, $t \equiv 2 \bmod 4, \mathcal{O}_{K}=\mathbb{Z}\left[\frac{1+\sqrt{d}}{2}\right]$, and 2 is inert in $\mathcal{O}_{K}$.

Considering the basis $\{\tau+t, 1\}$ of an $\mathcal{O}_{2 t}$-ideal with matrix

$$
P=\left(\begin{array}{ll}
1 & 0 \\
0 & 2
\end{array}\right)
$$

we obtain

$$
P\left(\begin{array}{c}
\tau+t \\
1
\end{array}\right)=[\tau+t, 2]
$$

as a basis of an $\mathcal{O}_{t}$-ideal. Applying Theorem $4.13(\mathrm{~b})$ yields that

$$
2^{-8} \frac{\Delta\left(\frac{\tau+1}{2}\right)}{\Delta(\tau)}
$$

is a unit, since $t \equiv 2 \bmod 4$ implies $l=1$ and $12\left(1-\left(1 / 2^{1-1} 3\right)\right)=8$, and thus by $(2-2)$ and $(2-1)$,

$$
2^{-8} \frac{\Delta\left(\frac{\tau+1}{2}\right)}{\Delta(\tau)}=\left(\frac{\mathfrak{f}^{3}}{2}\right)^{8}=\left(\frac{g(\tau)}{2}\right)^{8} .
$$

In the cases $m \equiv 11,19 \bmod 24$, the function $\mathfrak{f}(\tau)$ is a class invariant by Remark 3.6, since $\operatorname{gcd}(3, D(\tau))=1$. Hence in these cases, $g(\tau)$ has norm $2^{8 h_{t} / 24}$, which means that $l=h_{t} / 3$.

In the case $m \equiv 3 \bmod 24$, we have $3 \mid D(\tau)$, which means that $l=h_{t}$ for $\mathfrak{f}(\tau)^{3}$, implying that $\tilde{g}(\tau)=\mathfrak{f}(\tau)^{3} / 2$ is a class invariant and a unit.

For $m \equiv 4 \bmod 8$, we obtain

$$
d^{\prime} \equiv \begin{cases}3 \bmod 4 & \text { if } m \equiv 4 \bmod 16, \\ 1 \bmod 4 & \text { if } m \equiv 12 \bmod 16 .\end{cases}
$$


Now for $m \equiv 4 \bmod 16$, we get $\mathcal{O}_{K}=\mathbb{Z}\left[\sqrt{d^{\prime}}\right]$, that 2 ramifies in $\mathcal{O}_{K}$ with $t \equiv 2 \bmod 4$, and hence $l=1$. Applying Theorem $4.1(2(\mathrm{~b}))$ for

$$
P=\left(\begin{array}{ll}
1 & 0 \\
0 & 2
\end{array}\right)
$$

with the basis $\{\tau+t, 1\}$ of an $\mathcal{O}_{2 t}$-ideal, we obtain the unit

$$
2^{-9} \frac{\Delta\left(\frac{\tau}{2}\right)}{\Delta(\tau)}=\left(\left(\left(\frac{2}{A}\right) \frac{\mathfrak{f}_{1}(\tau)^{4}}{2 \sqrt{2}}\right)^{3}\right)^{2}=g(\tau)^{2},
$$

since $2^{12-\frac{6}{2^{\top}}}=2^{9}$, which shows that $g(\tau)$ is a unit in the case $m \equiv 4 \bmod 16$.

Lastly, for $m \equiv 12 \bmod 16$ with $d=d^{\prime} \equiv 1 \bmod 4$, we have

$$
\mathcal{O}_{K}=\mathbb{Z}\left[\frac{1+\sqrt{d^{\prime}}}{2}\right],
$$

and we have two different cases:

$$
d \equiv \begin{cases}1 \bmod 8, \text { i.e., } 2 \text { splits, } & \text { if } k \equiv 1,3,5 \bmod 6, \\ 5 \bmod 8, \text { i.e., } 2 \text { is inert, } & \text { if } k \equiv 0,2,4 \bmod 6 .\end{cases}
$$

Moreover, $s^{2} d \equiv 2 k \bmod 3, \quad$ which means that $\operatorname{gcd}(3, d)=1$ for $k \equiv 1,2,4,5 \bmod 6$ and hence together with Remark 3.6 that we can consider the invariants without the outer exponent 3. Applying analogously as above Theorem $4.1(3(\mathrm{~b}))$ for $k \equiv 0,2,4 \bmod 6$ and 4.1 (1(b)) for $k \equiv 1,3,5$ mod 6 together with Remark 3.6 for

$$
P=\left(\begin{array}{ll}
1 & 0 \\
0 & 2
\end{array}\right)
$$

with the basis $\{\tau+t, 1\}$ of an $\mathcal{O}_{2 t}$-ideal, we obtain the desired results.

Remark 4.5. For $l=2,3,5,7,11,13,17$ and $\tau \in \mathbb{H}$, the generalized Weber functions are defined as

$$
\mathfrak{w}_{l}:=\frac{\eta\left(\frac{\tau}{l}\right)}{\eta(\tau)} .
$$

(Note that $\mathfrak{w}_{l}=\mathfrak{f}_{1}$ for $l=2$.)

Using these functions, one can obtain new class invariants (see [Enge 07, pp. 15, 16] or [Gee and Stevenhagen 98, p. 450]). In [Uzunkol 10], the possibility of representing these class invariants as quotients of Thetanullwerte is discussed. Moreover, it is shown that most of these invariants are units using Theorem 4.1, and that better class invariants can be obtained in some cases as in Theorem 4.2.

\section{UNIT GROUP}

Due to the fact that the class invariants are units in most cases in the corresponding ring class fields by Theorems 4.2 and 4.4, we know the $h_{t}$ different units of the field $\Omega_{t}$, which is a totally complex field of absolute degree $2 h_{t}$. By Dirichlet's unit theorem, we know that the unit rank of the field $\Omega_{t}$ is $h_{t}-1$.

Since we know the conjugates of $g(\tau)$ explicitly, we can compute the full unit group if the $h_{t}-1$ conjugates of any $g(\tau)$ form a subgroup $U_{\Omega_{t}}$ of finite index of the unit group $E_{\Omega_{t}}$. In this case, one can obtain a larger subgroup $U_{\Omega_{t}}^{\prime}$ by checking whether certain elements are $p$ th powers; see [Hajir 88, Pohst and Zassenhaus 89] for the details. An upper bound $B$ for the index can be found by using a lower bound for the regulator of $\Omega_{t}$, which can be found using the algorithm in [Fieker and Pohst 08], which appears here as Algorithm 1.

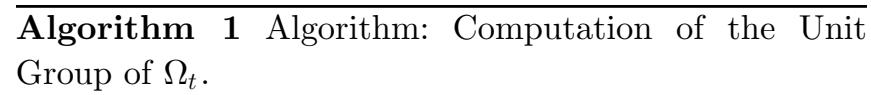

Input: An order $\mathcal{O}_{t}$ of an imaginary quadratic number field $K$ with conductor $t, \tau \in \mathcal{O}_{t}$, and $D(\tau)=-4 m$, where $m$ satisfies the congruence conditions of Theorems 4.2 and 4.4 , so that $g(\tau)$ is a unit.

Output: The generators of $E_{\Omega_{t}}$ of $\Omega_{t}$.

1. Find $h_{t}-1 \operatorname{roots} \epsilon_{1}, \ldots, \epsilon_{h_{t}-1}$ of $W_{D(\tau)}(x)$ such that the upper bound $B$ is minimal. If $B=\infty$ go to (5). If not go to (2).

2. List all prime numbers $p_{i} \leq B, i=1, \ldots, n$.

3. For $i=1$ to $n$,

(a) find the power $e_{i}$ of $p_{i}$, with the units $\gamma_{1}, \ldots, \gamma_{h_{t}-1}$ that form together with the roots of unity a subgroup of index $\left(E_{\Omega_{t}}: U_{\Omega_{t}}\right) / p_{i}^{e_{i}}$.

(b) Set $\epsilon_{1}, \ldots, \epsilon_{h_{t}-1}:=\gamma_{1}, \ldots, \gamma_{h_{t}-1}$.

4. Return the fundamental units $\gamma_{1}, \ldots, \gamma_{h_{t}-1}$ of $\Omega_{t}$.

5. Return The units form a subgroup of smaller rank.

In all examples we computed, we observed that the suitable $h_{t}-1$ conjugates of units obtained by Theorems 4.2 and 4.4 form a subgroup of finite index if $m \neq \equiv 5 \mathrm{mod}$ 8. Although the upper bound can become too large to use the above algorithm, this method is the most efficient one 
for computing the unit group of the corresponding ring class fields, since the units are explicitly known. We make the following conjecture.

Conjecture 5.1. If $m \not \equiv 5 \bmod 8$, the units constructed by Theorems 4.2 and 4.4 together with their conjugates form a subgroup of full rank of the corresponding unit group $E_{\Omega_{t}}$.

There are analogous results concerning elliptic units, which are the quotients of a $\Delta$-function (see [Hajir 88]). They form a subgroup of full rank.

Our numerical observations and the fact that elliptic units are mostly 24th powers of the units of Theorems 4.2 and 4.4 by equation (2-1) support the conjecture.

\section{EXAMPLES}

\subsection{Class Units}

Let $l$ be the largest absolute value of the coefficient of the class polynomial $W_{D}$ of $g(\tau)$ and let $l^{\prime}$ be the largest absolute value of the coefficient of the class polynomial $\tilde{W}_{D}$ of $g(\tau) / 2$ for the cases $m \equiv 3 \bmod 24$ and $m \equiv 4 \bmod$ 16 with $k \equiv 3 \bmod 6$ as in Theorem 4.4.
Let further $D=t^{2} d$ be the discriminant of the order $\mathcal{O}_{t}$ with $h_{D}:=h_{t}$. We obtained the results shown in Table 1 for $\gamma=l / l^{\prime}$ using MAGMA.

\section{2. $\theta$ versus $\eta$}

We compute the class polynomials using the invariants of Theorem 3.5 with a fixed precision for several discriminants. We obtained Table 2 using MAgmA. Moreover, the values of $\eta$-functions are computed using Theorem 3.11.

In the table, Prec denotes the fixed precision we used, and $q$ the quotient of the time required to compute the polynomials using eta representations by the time required to compute them using theta representations (given in seconds in the columns $\eta$ and $\theta$ respectively).

\subsection{Unit Group}

We consider the example $m=24 \cdot 3+3$. In this case the class polynomial, obtained from the new class invariant by Theorem 4.4 , is

$$
\tilde{W}_{-204}(x)=x^{6}-8 x^{5}-3 x^{4}+6 x^{3}+9 x^{2}+2 x+1 .
$$

Let $\tilde{g}(\tau)$ be the class invariant. Then the lower regulator bound $L=K(\tilde{g}(\tau))$, using the computer algebra

\begin{tabular}{lrccc}
\hline & $h_{D}$ & $l$ & $l^{\prime}$ & $\gamma$ \\
\hline-108 & 3 & 12 & 3 & 4.0 \\
-204 & 6 & 144 & 9 & 16.0 \\
-240 & 4 & 25464 & 6336 & 4.0 \\
-624 & 8 & 1935551872 & 181257400 & 10.68 \\
-684 & 12 & 86016 & 139 & 618.8201 \\
-1356 & 18 & $\leq 6114304$ & $\leq 6.5812$ & 3336.212 \\
-2544 & 20 & $\leq 3.47 \cdot 10^{24}$ & $4.98 \cdot 10^{15}$ & $\geq 67.68$ \\
-11496 & 36 & $\leq 2,3 \cdot 10^{66}$ & $\leq 3.92 \cdot 10^{47}$ & $\geq 5,63 \cdot 10^{17}$ \\
-59436 & 96 & $\leq 7.03 \cdot 10^{235}$ & $\leq 8.30 \cdot 10^{224}$ & $\geq 8.45 \cdot 10^{10}$ \\
-123888 & 104 & $\leq 1.98 \cdot 10^{2652}$ & $\leq 3.65 \cdot 10^{2555}$ & $\geq 5.41 \cdot 10^{96}$ \\
-4266864 & 1056 & $\leq 7.9 \cdot 10^{777}$ & $\leq 5.25 \cdot 10^{644}$ & $\geq 1.5 \cdot 10^{133}$ \\
-5867436 & 744 & $\leq 2 \cdot 10^{5308}$ & $\leq 3.4 \cdot 10^{5127}$ & $\geq 5.8 \cdot 10^{180}$ \\
-12677616 & 2000 & $\leq 2.6 \cdot 10^{7848}$ & $\leq 3.7 \cdot 10^{7626}$ & $\geq 7.1 \cdot 10^{221}$ \\
-45657072 & 2500 & $\leq 5.20 \cdot 10^{2346}$ & $6.79 \cdot 10^{1987}$ & $\geq 7.65 \cdot 10^{358}$ \\
-62506668 & 1992 & & & \\
\hline
\end{tabular}

TABLE 1. A comparison table for the coefficients. 


\begin{tabular}{lrrrrr}
\hline$D$ & $h_{D}$ & Prec & $\eta$ & $\theta$ & $q$ \\
\hline-104 & 6 & 8 & 0.04 & 0.01 & 4.0 \\
-260 & 8 & 12 & 0.05 & 0.01 & 5.0 \\
-684 & 12 & 40 & 0.09 & 0.02 & 4.5 \\
-1652 & 20 & 63 & 0.16 & 0.04 & 4.0 \\
-3740 & 28 & 85 & 0.25 & 0.07 & 3.57 \\
-14928 & 32 & 144 & 0.41 & 0.12 & 3.42 \\
-20904 & 40 & 147 & 0.46 & 0.12 & 3.83 \\
-39076 & 52 & 291 & 1.13 & 0.27 & 4.18 \\
-63372 & 96 & 393 & 2.43 & 0.56 & 4.34 \\
-77364 & 112 & 613 & 10.46 & 2.87 & 3.64 \\
-91068 & 122 & 467 & 5.66 & 1.34 & 4.22 \\
-107976 & 144 & 375 & 4.74 & 1.14 & 4.16 \\
-189744 & 168 & 664 & 15.68 & 3.87 & 4.05 \\
-1021732 & 292 & 1517 & 136.4 & 33.33 & 4.09 \\
\hline
\end{tabular}

TABLE 2. A comparison table for the different choices of representations of class invariants.

system $\mathrm{KANT} / \mathrm{KASH}^{1}$ is 43.3706 . Using the conjugate units $\tilde{g}^{(i)}$ for $i=1, \ldots, 5$, we compute $\operatorname{det}(\mathcal{R})=$ 74.6592. Hence, the upper bound for the index is $74.6592 / 43.3706=1.7214$, which means that the invariants are already fundamental units of $L=K(\tilde{g}(\tau))=$ $K(j(\tau))$.

\section{ACKNOWLEDGMENTS}

We thank the referee for a careful reading of the manuscript that led to considerable improvements in the quality of the paper.

\section{REFERENCES}

[Atkin and Morain 93] A. O. L. Atkin and F. Morain. "Elliptic Curves and Primality Proving." Math. Comp. 61 (1993), 29-67.

[Birch 69] B. Birch. "Weber's Class Invariants." Mathematik 16 (1969), 283-294.

[Blake et al. 99] I. Blake, G. Seroussi, and N. Smart. Elliptic Curves in Cryptography. Cambridge, UK: Cambridge University Press, 1999.

${ }^{1}$ Available online (http://www.math.tu-berlin.de/ kant/kash.
[Blake et al. 05] I. Blake, G. Seroussi, and N. Smart. Advances in Elliptic Curves in Cryptography. Cambridge, UK: Cambridge University Press, 2005.

[Bröker and Stevenhagen 08.] R. M. Bröker and P. Stevenhagen. "Constructing Elliptic Curves of Prime Order." In Computational Arithmetic Geometry, edited by K. E. Lauter and K. A. Ribet, Contemp. Math. 463, pp. 17-28. American Mathematical Society: Providence, RI, 2008.

[Deuring 58] M. Deuring. "Die Klassenkörper der komplexen Multiplikation." In Enzykl. d. math. Wiss., 2. Auflage, Heft 10. Stuttgart, 1958.

[Dupont 11] R. Dupont. "Fast Evaluation of Modular Functions Using Newton Iterations and the AGM." Math. Comp 80 (2011), 1823-1847.

[Enge 07] A. Enge. "Courbe Algébriques et Cryptologie." Habilitation, Université Paris, 2007. Available online (http://www.math.u-bordeaux1.fr/ enge/vorabdrucke/ Enge-Habil.pdf).

[Fieker and Pohst 08] C. Fieker and M. Pohst. "A Lower Regulator Bound for Number Fields." Journal of Number Theory 128 (2008), 2767-2775.

[Freeman et al. 06] D. Freeman, M. Scott, and E. Teske. "A Taxonomy of Pairing-Friendly Elliptic Curves." Available online (http://eprint.iacr.org/2006/372.pdf), 2006.

[Gee and Stevenhagen 98] A. Gee, P. Stevenhagen. "Generating Class Fields Using Shimura Reciprocity." LNCS 1423 (ANTS-III) (1998) 441-453.

[Hajir 88] F. Hajir. "Unramified Elliptic Units." PhD thesis, Princeton University, 1988.

[Hindry and Silverman 00] M. Hindry and J. Silverman. Diophantine Geometry: An Introduction. New York: Springer, 2000.

[Lang 73] S. Lang. Elliptic Functions. Reading, MA: AddisonWesley, 1973.

[Morain 07] F. Morain. "Implementing the Asymptotically Fast Version of the Elliptic Curve Primality Proving Algorithm." Math. Comp. 76 (2007), 493-505.

[Pohst and Zassenhaus 89] M. Pohst and H. Zassenhaus. Algorithmic Algebraic Number Theory. Cambridge, UK: Cambridge University Press, 1989.

[Sarnak 95] P. Sarnak. "Selberg's Eigenvalue Conjecture." Notices of the AMS 42 (1995), 1272-1277.

[Schertz 76] R. Schertz. "Die singulären Werte der Weberschen Funktionen $\mathfrak{f}, \mathfrak{f}_{1}, \mathfrak{f}_{2}, \gamma_{2}, \gamma_{3}$." J. Reine Angew. Math. $286 / 287$ (1976), 46-74. 
[Schertz 02] R. Schertz. "Weber's Class Invariants Revisited." Journal de Théorie des Nombres de Bordeaux 14 (2002), $325-343$.

[Uzunkol 10] O. Uzunkol. "Über die Konstruktion algebraischer Kurven mittels komplexer Multiplikation." $\mathrm{PhD}$ thesis, TU-Berlin, 2010.
[Weber 08] H. Weber. Lehrbuch der Algebra, Bd. 3, 2. Aufl. Braunschweig: Vieweg, 1908.

[Weng 01] A. Weng. "Konstruktion kryptographisch geeigneter Kurven mit komplexer Multiplikation." PhD thesis, Universität GH Essen, 2001.

Franck Leprévost, University of Luxembourg, 162 A, avenue de la Faïencerie, L-1511 Luxembourg (franck.leprevost@uni.lu)

Michael Pohst, TU-Berlin, Sekretariat MA 8-1, Straße des 17. Juni 136, D-10623 Berlin, Germany (pohst@mail.math.tu-berlin.de)

Osmanbey Uzunkol, Carl von Ossietzky Universität Oldenburg, Institut für Mathematik, D-26111, Oldenburg, Germany (osmanbey.uzunkol@uni-oldenburg.de)

Received October 1, 2009; accepted January 6, 2010. 